\title{
Cloud Computing and Its Advantages
}

\author{
Shaikh Ashapakh Sattar \\ Research Scholar, Dr. Babasaheb Ambedkar Marathwada University Aurangabad, India
}

\begin{abstract}
In this paper the concept of cloud computing and its advantages are explained. There is no doubt that businesses can reap huge benefits from cloud computing. Cloud Computing is a flexible, cost-effective, and proven delivery platform for providing business or consumer IT services over the Internet.
\end{abstract}

Keywords: Cloud, Public cloud, Advantages of cloud, Cloud services

\section{Introduction}

The importance of Cloud Computing is increasing and it is receiving a growing attention in the scientific and industrial communities. Cloud Computing enables ubiquitous, convenient, on-demand network access to a shared pool of configurable computing resources (e.g., networks, servers, storage, applications, and services) that can be rapidly provisioned and released with minimal management effort or service provider interaction

Cloud Computing appears as a computational paradigm as well as distribution architecture and its main objective is to provide secure, quick, convenient data storage and net computing service, with all computing resources visualized as services and delivered over the Internet. The cloud enhances collaboration, agility, scalability, availability, ability to adapt to fluctuations according to demand, accelerate development work, and provides potential for cost reduction through optimized and efficient computing.

\section{Types of Cloud}

Cloud computing is usually described in one of two ways, either based on the cloud location, or on the service that the cloud is offering. Based on a cloud location, it can classified as:

Public cloud: mean that the whole computing infrastructure is located on the premises of a cloud computing company that offers the cloud service. The location remains, thus, separate from the customer and he has no physical control over the infrastructure. As public clouds use shared resources, they do excel mostly in performance, but are also most vulnerable to various attacks.

Private cloud means using a cloud infrastructure (network) solely by one customer/organization. It is not shared with others, yet it is remotely located. If the cloud is externally hosted. The companies have an option of choosing an onpremise private cloud as well, which is more expensive, but they do have a physical control over the infrastructure. The security and control level is highest while using a private network. Yet, the cost reduction can be minimal, if the company needs to invest in an on-premise cloud infrastructure.

\section{Private clouds}

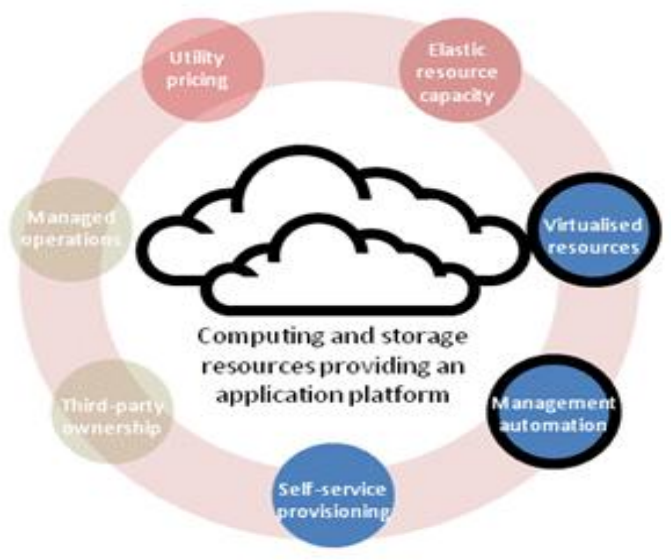

Figure 1: Private Cloud

Public clouds

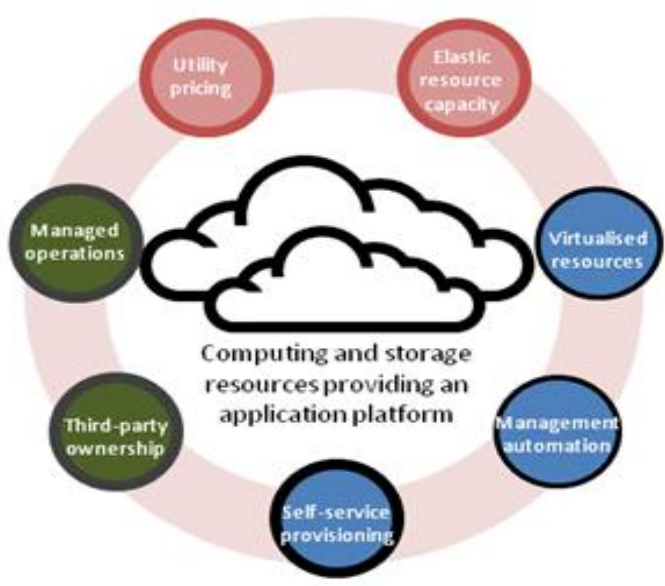

Figure 2: Public Cloud

Hybrid cloud, of course, means, using both private and public clouds, depending on their purpose. For example, public cloud can be used to interact with customers, while keeping their data secured through a private cloud. 


\section{International Journal of Science and Research (IJSR) \\ ISSN (Online): 2319-7064}

Index Copernicus Value (2013): 6.14 | Impact Factor (2015): 6.391

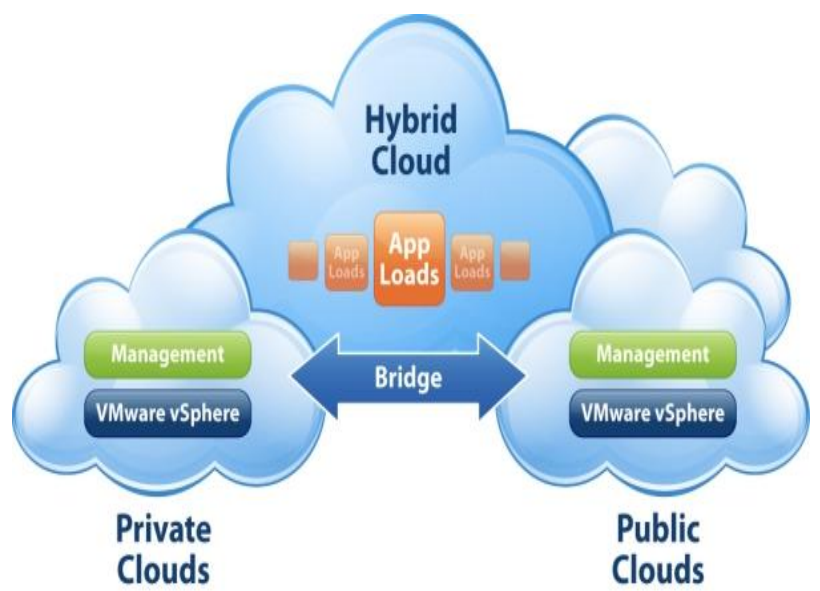

Figure 3: Hybrid Cloud

Community cloud implies an infrastructure that is shared between organizations, usually with the shared data and data management concerns. For example, a community cloud can belong to a government of a single country. Community clouds can be located both on and off the premises.

Based on a service that the cloud is offering, we are speaking of either:

IaaS (Infrastructure-as-a-Service) The most common cloud service is that one offering data storage disks and virtual servers, i.e. infrastructure. Examples of Infrastructure-as-a-Service (IaaS) companies are Amazon, Rackspace, Flexiscale.

PaaS (Platform-as-a-Service) the model offers a development platform, and this includes operating system, programming language execution environment, database, and web server. Operating system can be frequently upgraded and developed with PaaS, services can be obtained from diverse sources, and programming can be worked in teams (geographically distributed)

SaaS (Software-as-a-Service) means that users can access various software applications on a pay-per-use basis. As opposed to buying licensed programs, often very expensive.

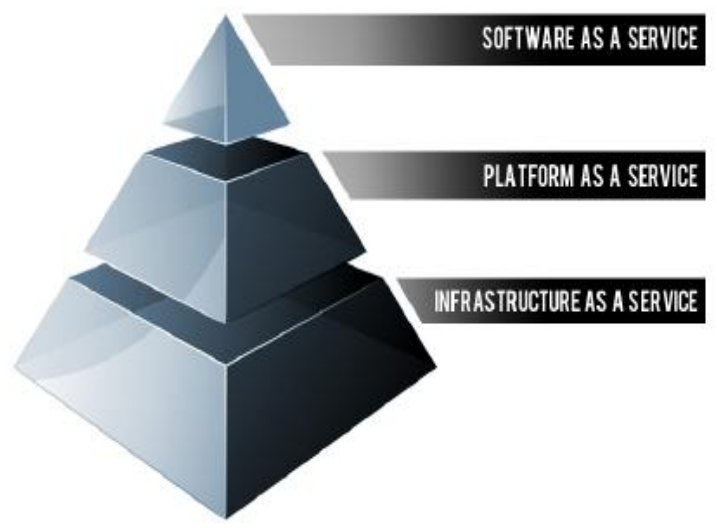

Figure 4: Cloud Service Models

Previously, we have explained how cloud works. Basically, programs that are needed to run a certain application are now more popularly located on a remote machine, owned by another company. This is done in order not to lose on the quality performance due to processing power of your own computer, to save money on IT support, and yet remain advantageous on the market. These computers that run the applications, store the data, and use a server system, are basically what we call "the cloud".

\section{Advantages of Cloud Computing}

a) Cost Efficiency: A big advantage of cloud computing is the removing the investment in software or servers. By leveraging the cloud's capabilities, companies can save on licensing fees and, at the same time, eliminate overhead charges, such as the cost of data storage, software updates, management, etc. With the cloud, it is possible to set up one-time-payments or pay-as-you-go to help save on costs that can add up when using a traditional IT model. A public-cloud delivery model converts capital expenditure to operational expenditure. Here is a better look at some of the areas where cloud computing can save money:

b) Servers: There is no longer a need for owning/buying servers with cloud computing. Using the cloud, you are leasing a virtual server instead of a physical one. Your data is stored in a virtual space that can even be paid for by the hour, meaning you can pay for the space only when you need it.

c) Employees: The cloud cuts, or at least reduces the need head count for an in-house IT staff. Using the cloud allows you to do away with manual updates. Instead of doling out work to a system administrator, who then manually completes the task and makes the resource available, cloud computing uses resource APIs and an orchestration engine to drive the same task. This is the genius of cloud computing.

d) Maintenance and Downtime: Cloud outages do happen, sure, but as cloud technology matures, outages have become less common. Cloud providers are able to actively monitor, maintain and upgrade your hosting servers so you don't have to. The decrease in downtime means that you are not held back from business productivity.

\section{Location Independence and Continuous Availability}

Public clouds offer services that are available wherever the end user might be located. There are no longer physical boundaries related to server location and storage. The shackles are off. Data storage, computing and networking can all be done in an independent location. This method enables easy access to information and accommodates the needs of users in different time zones and geographic locations. Traditionally, on-site servers create an island of infrastructure. Infrastructure that exists at data centers in remote offices, at home, in billions of mobile devices and in the private cloud. Trying to manage all of these islands is a full time job. A major benefit of the cloud is readilyavailable information, resulting in collaboration booms, since it is now easier than ever to access, view and modify shared documents and files. 


\section{Mobility}

One of the main advantages of working in the cloud is that it allows users the mobility necessary in this day and age of global marketing. For example, a busy executive on a business trip in Japan may need to know what is going on at the company headquarters in Australia. Rather than having to make phone calls and rely on the reports of employees, they can simply check updated statistics online. The Internet is, for the most part, everywhere. Therefore, cloud computing allows the mobility necessary for success.

\section{Only Pay for What You Need}

Unlike many computing programs where the package comes with unnecessary applications, the cloud allows users to literally get what they pay for. This scalability allows for you to simply purchase the applications and data storage you really need.

\section{Backup and Recovery}

Since data now resides on the cloud and not on a local physical device. The various cloud providers offer reliable and flexible backup/recovery solutions. In some cases, the cloud itself is used solely as a backup repository of the data located in local computers. A few of the benefits of data backup in the cloud are listed below

a) Redundant Backups: Your data is always stored off-site and is redundantly copied to other servers in different locations. If one goes down, your data can be retrieved from elsewhere on the backup network.

b) Security: Data is encrypted by the backup service's software program on your computer before it is sent to the cloud, so thieves on the Internet can't intercept it.

c) Virus Protection: The backup service software detects any virus or infection before data is sent. If a virus is found, that file is flagged and not copied to the backup service. You will be notified that the corrupted file has not been deleted from your computer, so you won't lose any data, but it won't be backed up online.

\section{Conclusion}

Cloud computing provide different types of services with wide range of choices. One can select the type of service which suits to their organization and reduce the IT and operational cost. More over organizations need not to worry of buying/renewing the software license. All cloud providers provide high availability, data backups for better business continuity planning protection from security threads like virus. Adapting cloud provides better flexibility to access application and data as data and applications are saved in cloud. It can be accessed over internet from anywhere irrespective of geographic location.

\section{References}

[1] http://jisajournal.springeropen.com/articles/10.1186/186 9-0238-4-5

[2] http://www.infoworld.com/article/3041078/security/thedirty-dozen-12-cloud-security-threats.html

[3] http://www.modgility.com/cloud-computingadvantages-and-disadvantages/ 